\title{
Resonance phenomenon related to spectral singularities, complex barrier potential, and resonating waveguides
}

\author{
Ali Mostafazadeh* \\ Department of Mathematics, Koç University, Sarlyer, 34450 Istanbul, Turkey
}

(Received 8 May 2009; revised manuscript received 16 July 2009; published 25 September 2009)

\begin{abstract}
A peculiar property of complex scattering potentials is the appearance of spectral singularities. These are energy eigenvalues for certain scattering states that similarly to resonance states have infinite reflection and transmission coefficients. This property reveals an interesting resonance effect with possible applications in waveguide physics. We study the spectral singularities of a complex barrier potential and explore their application in designing a waveguide that functions as a resonator. We show that for the easily accessible sizes of the waveguide and its gain region, we can realize the spectral singularity-related resonance phenomenon at almost every wavelength within the visible spectrum or outside it.
\end{abstract}

DOI: 10.1103/PhysRevA.80.032711

PACS number(s): 03.65.Nk, 42.25.Bs, 24.30.Gd

\section{INTRODUCTION}

Recently it has been noticed that by defining the Hilbert space of a quantum system using a nonstandard inner product one can generate a unitary time-evolution by a Hamiltonian operator that is manifestly non-Hermitian in the standard $L^{2}$-inner product [1]. A typical example is $H$ $=-\left(d^{2} / d x^{2}\right)+i x^{3}$. It turns out that one can actually describe the corresponding physical system using the standard Hilbert space and a Hermitian Hamiltonian which is much more complicated and highly nonlocal [2]. The non-Hermitian Hamiltonians with the above property must have a real spectrum and a complete set of eigenfunctions so that every wave function can be expanded in terms of these. There are two main mechanisms that are responsible for the incompleteness of the eigenfunctions of a non-Hermitian Hamiltonian. These are associated with the presence of exceptional points [3] and spectral singularities [4].

An exceptional point is a point in the space of parameters $M$ of the Hamiltonian operator $H$ where an eigenvalue of $H$ becomes defective, i.e., if the parameters change continuously along a path in $M$ that passes through an exceptional point, two or more of the eigenvalues of $H$ together with their eigenvectors coalesce. Exceptional points have found various physical realizations and applications [5]. They have also been the subject of experimental studies [6]. In contrast, spectral singularities are certain points of the continuous spectrum of non-Hermitian scattering Hamiltonians [7] whose presence ruins the completeness of the eigenfunctions, although for each point of the spectrum (including the spectral singularity) there correspond two linearly independent eigenfunctions [8]. The physical meaning and importance of this strange mathematical phenomenon have been obscure until very recently [9].

The appearance of spectral singularities as an obstruction for defining a unitary quantum system using a complex potential was initially noted by Samsonov [10] who, following the pioneering work of Naimark $[4,11]$, only considered systems defined on the half-line. A simple example of an exactly

\footnotetext{
*amostafazadeh@ku.edu.tr
}

solvable model defined on the whole real line that can support a spectral singularity is the one given by a delta-function potential, $v(x)=\mathfrak{z} \delta(x)$, with a complex coupling constant $\mathfrak{z}$. As shown in [12] this model has a spectral singularity provided that $\mathfrak{z}$ is purely imaginary. The emergence and the mechanism by which a spectral singularity spoils the completeness of the eigenfunctions of a scattering non-Hermitian Hamiltonian are studied in [8].

In Ref. [9] we have provided a physical interpretation for the spectral singularities that identifies them with the energies of scattering states having infinite reflection and transmission coefficients. Because this is a characteristic property of resonance states, spectral singularities correspond to the resonance states having a real energy and hence a zero width. The existence of such states reveals a new type of resonance effect with potential applications in various areas of physics. To demonstrate how one can realize this effect, we have explored, in Ref. [9], the occurrence of spectral singularities for the $\mathcal{P} \mathcal{T}$-symmetric imaginary barrier potential,

$$
v(x):= \begin{cases}-i \zeta \operatorname{sgn}(x) & \text { for }|x|<\alpha \\ 0 & \text { for }|x| \geq \alpha,\end{cases}
$$

where $\zeta$ and $\alpha$ are real parameters, $\alpha$ is positive, and $\operatorname{sgn}(x)$ stands for the sign of $x$ [13].

The potential (1) can be used to model the propagation of certain transverse electric (TE) fields in a planar slab waveguide with an adjacent pair of loss and gain regions [14], as shown in Fig. 1. In [9], we have determined the values of physical parameters of this system that correspond to a spectral singularity. It turns out that for fixed values of the pa-

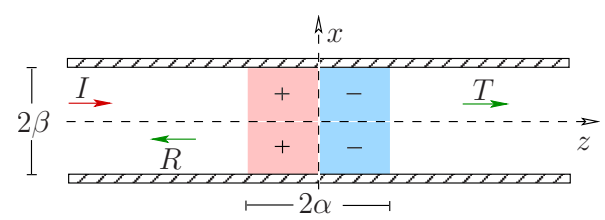

FIG. 1. (Color online) Cross section of a rectangular waveguide with gain $(+)$ and loss (-) regions in the $x-z$ plane. Arrows labeled by $I, R$, and $T$ represent the incident, reflected, and transmitted waves. 
rameters entering the permittivity, spectral singularities occur for a discrete set of values for the length of the loss and gain regions $2 \alpha$ and the height of the waveguide $2 \beta$. For the fundamental mode of the standing wave formed in the transverse direction (along the $x$ axis), the largest allowed values of $\alpha$ and $\beta$ are 1004.17 and $62.0464 \mathrm{~nm}$, respectively. Explicit calculation shows that for these values of $\alpha$ and $\beta$, the reflection and transmission coefficients (at the resonance frequency) exceed $10^{14}$. Note however that this requires maintaining this value of $\alpha$ for the length of the loss and gain regions to an extremely high degree of precision $\left(10^{-1} \AA\right)$. A $0.1 \%$ deviation from the above values of $\alpha$ and $\beta$ leads to a decrease in the reflection and transmission coefficients by a factor of $10^{7}$. Furthermore, the experimental realization of spectral singularities that is proposed in [9] involves restricting the frequency of propagating wave to the resonance frequency of the active region and demands that the imaginary part of the permittivity for the loss and gain differs only by a sign.

In the present paper, we study the spectral singularities of the complex barrier potential

$$
v(x):= \begin{cases}\mathfrak{z} & \text { for }|x|<\alpha \\ 0 & \text { for }|x| \geq \alpha,\end{cases}
$$

where $\mathfrak{z}$ is an arbitrary complex coupling constant. Our main motivation is that the proposed experimental setup that would confirm the resonance phenomenon associated with the spectral singularities of Eq. (2) is free of most of the above-mentioned restrictions. This is essential because of the additional degree of freedom, namely, the real part of $\mathfrak{z}$ in Eq. (2). Another reason for considering the potential (2) is that, similarly to (1), the mathematical problem of locating the spectral singularities of (2) admits an exact and essentially analytic solution. This is very convenient, for it enables us to avoid dealing with the subtleties of the numerical treatments of the problem.

A most remarkable result of our investigation is that for the experimentally easily accessible dimensions of the waveguide associated with Eq. (2), we can produce the spectral singularity-related resonance effect at almost every wavelength within the visible spectrum or outside it. This is of great practical significance for it may form the basis of a new mechanism for producing laser beams of desired wavelength.

This paper is organized as follows. In Sec. II, we provide a brief review of the results of $[8,9]$ on the calculation of spectral singularities and their relation to the reflection and transmission coefficients. In Sec. III, we solve the problem of locating the spectral singularities of the complex barrier potential. In Sec. IV, we describe a waveguide modeled using this potential and explore the possibility of the detection of the resonance effect associated with the spectral singularities. In Sec. V, we present our concluding remarks.

\section{SPECTRAL SINGULARITIES}

Consider the Hamiltonian operator $H=-\left(d^{2} / d x^{2}\right)+v(x)$ for a complex scattering potential $v(x)$ with $x \in \mathbb{R}$. Suppose that $v(x)$ tends to zero as $x \rightarrow \pm \infty$ so rapidly that the integral $\int_{-\infty}^{\infty}(1+|x|)|v(x)| d x$ converges. Then the spectrum of $H$ has a real continuous part. Suppose for simplicity that the spectrum coincides with $[0, \infty)$. Then the eigenvalue equation $H \psi=E \psi$ yields the following asymptotic expressions for the (generalized) eigenfunctions of $H$ :

$$
\psi_{k}^{\mathfrak{g}}(x) \rightarrow A_{ \pm}^{\mathfrak{g}} e^{i k x}+B_{ \pm}^{\mathfrak{g}} e^{-i k x} \text { for } x \rightarrow \pm \infty .
$$

Here $\mathfrak{g}$ is a degeneracy label taking values 1 and $2, k:=\sqrt{E}$, and $A_{ \pm}^{\mathfrak{g}}, B_{ \pm}^{\mathfrak{g}}$ are possibly $k$-dependent complex coefficients that are related by the so-called transfer matrix $\mathbf{M}=\left(M_{i j}\right)$ according to

$$
\left(\begin{array}{c}
A_{+}^{\mathfrak{g}} \\
B_{+}^{\mathfrak{g}}
\end{array}\right)=\mathbf{M}\left(\begin{array}{c}
A_{-}^{\mathfrak{g}} \\
B_{-}^{\mathfrak{g}}
\end{array}\right) .
$$

The transfer matrix encodes all the necessary information about the scattering properties of the system. It is easy to show that it has a unit determinant [8].

Next, consider the Jost solutions $\psi_{k \pm}$ of the eigenvalue equation $H \psi=k^{2} \psi$ that by definition satisfy

$$
\psi_{k \pm}(x) \rightarrow e^{ \pm i k x} \text { for } x \rightarrow \pm \infty .
$$

Denoting the coefficients $A_{ \pm}^{\mathfrak{g}}$ and $B_{ \pm}^{\mathfrak{g}}$ for the Jost solutions $\psi_{k \pm}$ by $A_{ \pm}^{ \pm}$and $B_{ \pm}^{ \pm}$and using of Eqs. (3)-(5), we find $A_{+}^{+}$ $=B_{-}^{-}=1, A_{-}^{-}=B_{+}^{+}=0, A_{-}^{+}=B_{+}^{-}=M_{22}, A_{+}^{-}=M_{12}$, and $B_{-}^{+}=-M_{21}$ [8]. These relations identify $\psi_{k \pm}$ with the left- and rightgoing scattering solutions [15]. As a result, the left and right transmission $T^{l, r}$ and reflection $R^{l, r}$ amplitudes are given by [9]

$$
T^{l}=T^{r}=\frac{1}{M_{22}}, \quad R^{l}=-\frac{M_{21}}{M_{22}}, \quad R^{r}=\frac{M_{12}}{M_{22}} .
$$

Spectral singularities of $H$ are eigenvalues $E=k^{2}$ for which $\psi_{k+}$ and $\psi_{k-}$ become linearly dependent $[4,16,17]$. They are given by the real zeros of $M_{22}$ where both the transmission and reflection coefficients diverge [9]. Because the latter is a characteristic feature of resonances [18], we associate spectral singularities with a peculiar type of resonances which satisfy the eigenvalue equation $H \psi=k^{2} \psi$ for a real $k^{2}$. These are resonances having a zero width [9].

\section{COMPLEX BARRIER POTENTIAL}

Consider the complex barrier potential (2). The solution of the time-independent Schrödinger equation, $H \psi=k^{2} \psi$, for this potential is elementary. We can use this solution to determine the entries of the transfer matrix $\mathbf{M}$,

$$
\begin{gathered}
M_{11}=\frac{e^{-2 i \alpha k} f(w,-\alpha k)}{4 w}, \\
M_{12}=-M_{21}=\frac{i\left(w^{2}-1\right) \sin (2 \alpha k w)}{2 w}, \\
M_{22}=\frac{e^{2 i \alpha k} f(w, \alpha k)}{4 w},
\end{gathered}
$$

where $w:=\sqrt{1-\mathfrak{z} / k^{2}}[19]$, and for all $\chi \in \mathbb{R}$, 


$$
f(w, \chi):=e^{-2 i \chi w}(1+w)^{2}-e^{2 i \chi w}(1-w)^{2} .
$$

According to Eq. (9), spectral singularities are associated with real values of $k$ for which

$$
f(w, \alpha k)=f\left(\sqrt{1-\mathfrak{z} / k^{2}}, \alpha k\right)=0 .
$$

This is a complex transcendental equation involving four real variables $\alpha, k, \operatorname{Re}(\mathfrak{z})$, and $\operatorname{Im}(\mathfrak{z})$, where "Re" and "Im" stand for the real and imaginary parts of their argument. A direct numerical solution of Eq. (11) would amount to identifying two of the variables as parameters $[\operatorname{say} \operatorname{Re}(\mathfrak{z})$ and $\operatorname{Im}(\mathfrak{z})]$ and solving Eq. (11) for the remaining two variables (i.e., $\alpha$ and $k)$. This requires selecting a range of values for the parameters and performing a numerical solution of Eq. (11) for all the values of the parameters within this range. This is not a straightforward procedure for in principle there is a real solution only for a one-dimensional subset of the twodimensional parameter space. Numerical treatments may miss part of this one-dimensional subset and could lead to various types of errors [20]. In the following, we shall avoid these difficulties by offering an exact and essentially analytic solution of Eq. (11). Specifically, we shall reduce Eq. (11) to a single real transcendental equation involving two real variables and a discrete label. As we will see this provides a much more efficient and precise method of locating spectral singularities of the potential (2).

We begin our analysis by recalling that in light of Eq. (10), we can express Eq. (11) in the form

$$
e^{i \alpha k w}(1-w)= \pm e^{-i \alpha k w}(1+w) .
$$

This is equivalent to

$$
\cos \left(2 \alpha k \sqrt{1-\mathfrak{z} / k^{2}}\right)= \pm\left(\frac{2 k^{2}}{\mathfrak{z}}-1\right) .
$$

We can reduce Eq. (13) into a pair of real equations. To this end, we first introduce

$$
\begin{gathered}
\rho:=\frac{\operatorname{Re}(\mathfrak{z})}{k^{2}}, \quad \sigma:=\frac{\operatorname{Im}(\mathfrak{z})}{k^{2}}, \quad y:=\frac{\sigma}{1-\rho}, \\
q:=\alpha k \sqrt{2|1-\rho|\left(\sqrt{y^{2}+1}-1\right)} \operatorname{sgn}(y), \\
r:=\alpha k \sqrt{2|1-\rho|\left(\sqrt{y^{2}+1}+1\right)} .
\end{gathered}
$$

In light of Eqs. (15) and (16) and $k>0$, we have

$$
\operatorname{sgn}(q)=\operatorname{sgn}(y) \text { and } \quad r>0 .
$$

Next, by inserting $\mathfrak{z} / k^{2}=\rho+i \sigma$ into Eq. (13) and using the elementary properties of the trigonometric and hyperbolic functions, we express the real and imaginary parts of this equation as

$$
\cos r \cosh q= \pm \frac{1-(1-\rho)^{2}\left(y^{2}+1\right)}{(1-\rho)^{2} y^{2}+\rho^{2}},
$$

$$
\sin r \sinh q=\mp \frac{2(1-\rho) y}{(1-\rho)^{2} y^{2}+\rho^{2}} .
$$

Here either the top or the bottom sign should be taken in both of the equations.

We can easily eliminate $r$ in Eqs. (18) and (19). We do this by solving for $\cos r$ and $\sin r$ and imposing the identity $\sin ^{2} r+\cos ^{2} r=1$. Expressing $\cosh ^{2} q$ that appears in the resulting equation as $1+\sinh ^{2} q$, we find after some lucky cancellations

$$
\begin{aligned}
& \sinh ^{4} q-\left(\frac{4(1-\rho)}{(1-\rho)^{2} y^{2}+\rho^{2}}\right) \sinh ^{2} q-\left(\frac{4(1-\rho)^{2} y^{2}}{\left[(1-\rho)^{2} y^{2}+\rho^{2}\right]^{2}}\right) \\
& \quad=0 .
\end{aligned}
$$

Solving this equation for $q$ and using Eq. (17), we obtain

$$
q=\operatorname{sgn}(y) Q(\rho, y),
$$

where

$$
Q(\rho, y):=\sinh ^{-1}\left[\sqrt{\frac{2\left(\sqrt{y^{2}+1}|1-\rho|+1-\rho\right)}{(1-\rho)^{2} y^{2}+\rho^{2}}}\right] .
$$

Next, we substitute Eq. (20) in Eq. (18) and solve for $r$. Employing the identity $\cosh \left[\sinh ^{-1}(x)\right]=\sqrt{1+x^{2}}$ to simplify the resulting expression, we then find

$$
r=R_{n}^{\epsilon}(\rho, y),
$$

where $n$ is an integer, $\epsilon$ is a sign, and

$$
R_{n}^{ \pm}(\rho, y):=\pi n \pm \cos ^{-1}\left(\frac{1-|1-\rho| \sqrt{y^{2}+1}}{\sqrt{(1-\rho)^{2} y^{2}+\rho^{2}}}\right) .
$$

Three remarks are in order.

(1) Because for real values of $\rho$ and $y$, we have

$$
|1-\rho| \sqrt{y^{2}+1}+\rho-1 \geq 0,
$$

and

$$
\begin{aligned}
& (1-\rho)^{2} y^{2}+\rho^{2} \\
& \quad=\left(1-|1-\rho| \sqrt{y^{2}+1}\right)^{2}+2\left(|1-\rho| \sqrt{y^{2}+1}+\rho-1\right),
\end{aligned}
$$

the argument of $\cos ^{-1}$ in Eq. (23) lies in the interval $[-1,1]$. This implies that the second term on the right-hand side of Eq. (23) takes values in $[0, \pi]$, and $R_{n}^{ \pm}(\rho, y)$ is always real. Furthermore, $\operatorname{sgn}\left[R_{n}^{ \pm}(\rho, y)\right]=\operatorname{sgn}(n)$ for $n \neq 0$ and $\operatorname{sgn}\left[R_{0}^{ \pm}(\rho, y)\right]= \pm$ except for the case $\rho=y=0$ which corresponds to a real barrier potential. Therefore, to ensure that $r>0$, we must demand that either $n>0$ and $\epsilon$ be arbitrary or $n=0$ and $\epsilon=+$. In the following we shall confine our attention to these two cases.

(2) Every $r$ and $q$ that satisfy Eqs. (18) and (19) must also satisfy Eqs. (20) and (22), but the converse is not true. It turns out that depending on the choice of the top or bottom sign in Eqs. (18) and (19) and range of values of $\rho$ and $y$ one must further restrict the values of $n$ and $\epsilon$ in Eq. (22).

(3) Equations (18) and (19) are not equivalent to Eq. (13) unless we impose Eqs. (15) and (16). First, we impose Eq. 
(16). This allows us to express $\alpha k$ in terms of $r, \rho$, and $y$. In view of Eq. (22), we then find

$$
\alpha k=G_{n}^{\epsilon}(\rho, y):=\frac{R_{n}^{\epsilon}(\rho, y)}{\sqrt{2|1-\rho|\left(\sqrt{y^{2}+1}+1\right)}} .
$$

Unlike Eq. (16) that determines $\alpha k$ in terms of $\rho$ and $y$, Eq. (15) gives rise to an additional constraint on the possible values of $\rho$ and $y$. We will next derive an explicit form of this constraint.

We can use Eqs. (15) and (16) to obtain

$$
q=r \sqrt{\frac{\sqrt{y^{2}+1}-1}{\sqrt{y^{2}+1}+1}} \operatorname{sgn}(y) .
$$

If we insert Eq. (22) in this equation and use Eq. (17), we arrive at

$$
q=\operatorname{sgn}(y) \widetilde{Q}_{n}^{\epsilon}(\rho, y),
$$

where

$$
\tilde{Q}_{n}^{\epsilon}(\rho, y):=R_{n}^{\epsilon}(\rho, y) \sqrt{\frac{\sqrt{y^{2}+1}-1}{\sqrt{y^{2}+1}+1}} .
$$

Recall that here $n$ is a non-negative integer and for $n=0$ we have $\epsilon=+$.

Enforcing Eqs. (20) and (28) yields $Q(\rho, y)=\widetilde{Q}_{n}^{\epsilon}(\rho, y)$. This gives an infinite sequence of equations that determine the locus of points in the $\rho-y$ plane for which the complex barrier potential has a spectral singularity. These form an infinite sequence of curves. Clearly, $Q(\rho, y)=\widetilde{Q}_{n}^{\epsilon}(\rho, y)$ is equivalent to $F_{n}^{\epsilon}(\rho, y)=0$, where

$$
F_{n}^{\epsilon}(\rho, y):=\frac{1}{2}\left[\sinh ^{2} Q(\rho, y)-\sinh ^{2} \widetilde{Q}_{n}^{\epsilon}(\rho, y)\right] .
$$

In view of Eqs. (21), (29), and (30) we have the following explicit expression for $F_{n}^{\epsilon}$ :

$$
\begin{aligned}
F_{n}^{ \pm}(\rho, y)= & \frac{|1-\rho| \sqrt{y^{2}+1}+1-\rho}{(1-\rho)^{2} y^{2}+\rho^{2}}-\frac{1}{2} \sinh ^{2}\left\{\sqrt{\frac{\sqrt{y^{2}+1}-1}{\sqrt{y^{2}+1}+1}}\right. \\
& \left.\times\left[\pi n \pm \cos ^{-1}\left(\frac{1-|1-\rho| \sqrt{y^{2}+1}}{\sqrt{(1-\rho)^{2} y^{2}+\rho^{2}}}\right)\right]\right\} .
\end{aligned}
$$

In the $\rho-\sigma$ plane, which coincides with the complex $\mathfrak{z} / k^{2}$ plane, the spectral singularities are located on the curves

$$
C_{n}^{\epsilon}:=\left\{(\rho, \sigma) \in \mathbb{R}^{2} \mid F_{n}^{\epsilon}\left(\rho, \frac{\sigma}{1-\rho}\right)=0\right\} .
$$

As we will see below, not all the points on these curves turn out to correspond to a spectral singularity.

Examining the graphs of $C_{n}^{\epsilon}$ (see Fig. 2), we find that these curves all lie to the left of the line: $\rho=1$ in the $\rho-\sigma$ plane. The curve $C_{0}^{+}$intersects this line at $(\rho=1, \sigma=0)$ but this point does not correspond to a spectral singularity because for $\sigma=0$ the potential is real. These observations allow us to confine our attention to the case $\rho<1$. A consequence of this relation and Eq. (17) is

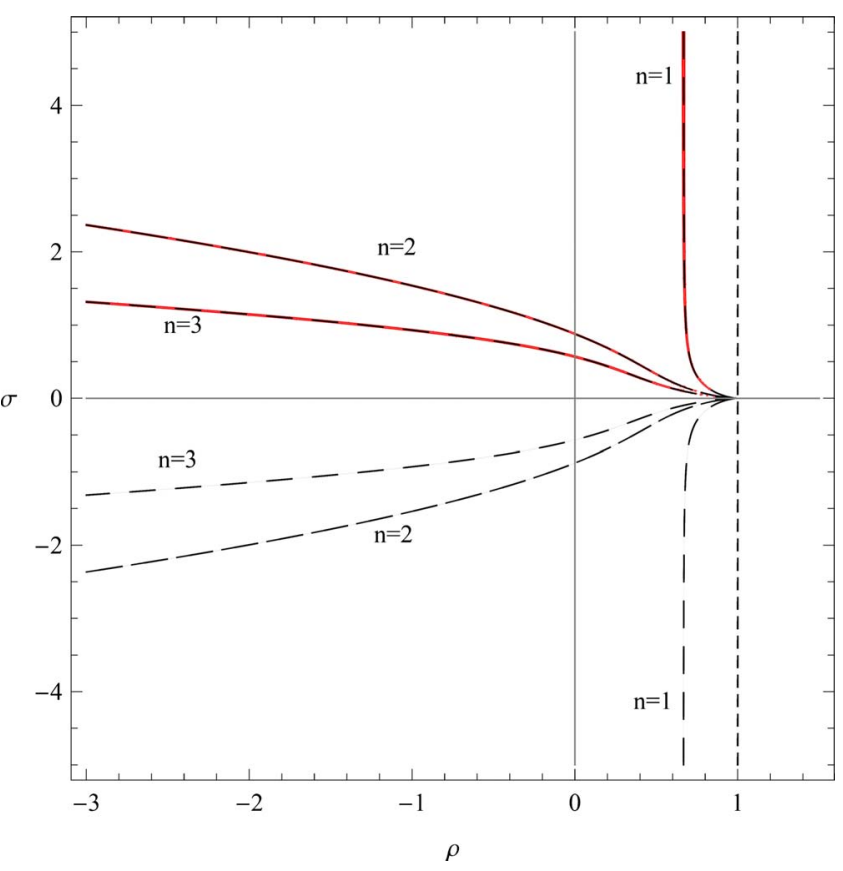

FIG. 2. (Color online) Graphs of the curves $C_{n}^{-}$(the dashed black curves) and $\widetilde{C}_{n}^{-}$(the solid red curves) in the $\rho-\sigma$ plane for $n$ $=1,2,3 . C_{n}^{-}$are symmetric about the $\rho$ axis. $\widetilde{C}_{n}^{-}$coincides with $C_{n}^{-}$ for $\sigma>0$. This shows the lack of spectral singularities for $\sigma \leq 0$. The dashed black line corresponds to $\rho=1$. The solid gray lines are the coordinate axes.

$$
\operatorname{sgn}(q)=\operatorname{sgn}(y)=\operatorname{sgn}(\sigma)=\operatorname{sgn}[\operatorname{Im}(\mathfrak{z})] .
$$

In order to determine the choices of $n$ and $\epsilon$ for which $C_{n}^{\epsilon}$ includes points corresponding to spectral singularities, we proceed as follows. First, we recall that the spectral singularities are give by Eq. (11). Next, we use the identities $w$ $=\sqrt{1-\rho-i \sigma}=\sqrt{1-\rho-i(1-\rho) y}$ and Eqs. (10) and (26) to express the left-hand side of Eq. (11) in terms of $\rho$ and $y$. This yields a sequence of equations of the form $\widetilde{F}_{n}^{\epsilon}(\rho, y)=0$, where

$$
\begin{aligned}
\widetilde{F}_{n}^{\epsilon}(\rho, y):= & e^{-2 i G_{n}^{\epsilon}(\rho, y) \sqrt{1-\rho-i(1-\rho) y}}[1+\sqrt{1-\rho-i(1-\rho) y}]^{2} \\
& -e^{2 i G_{n}^{\epsilon}(\rho, y) \sqrt{1-\rho-i(1-\rho) y}}[1-\sqrt{1-\rho-i(1-\rho) y}]^{2},
\end{aligned}
$$

where $G_{n}^{\epsilon}$ is introduced in Eq. (26). The spectral singularities lie on the curves

$$
\widetilde{C}_{n}^{-}:=\left\{(\rho, \sigma) \in \mathbb{R}^{2}|| \widetilde{F}_{n}^{\epsilon}\left(\rho, \frac{\sigma}{1-\rho}\right) \mid=0\right\} .
$$

It turns out that $\left|\tilde{F}_{n}^{\epsilon}\left(\rho, \frac{\sigma}{1-\rho}\right)\right|=0$ only for $\epsilon=-$ and $n>0$. Furthermore, as shown in Fig. 2, the graph of $\widetilde{C}_{n}^{-}$coincides with a part of the graph of $C_{n}^{-}$that lies above the $\rho$ axis in the $\rho-\sigma$ plane. That is,

$$
\widetilde{C}_{n}^{-}=\left\{(\rho, \sigma) \in \mathbb{R}^{2} \mid F_{n}^{-}\left(\rho, \frac{\sigma}{1-\rho}\right)=0, \sigma>0\right\} .
$$

This provides a highly nontrivial check on the validity of our calculations. It also reveals the interesting fact that spectral singularities can appear only for the cases that the coupling constant $\mathfrak{z}$ has a positive imaginary part. In Sec. IV, we will provide a simple physical justification for this fact. 


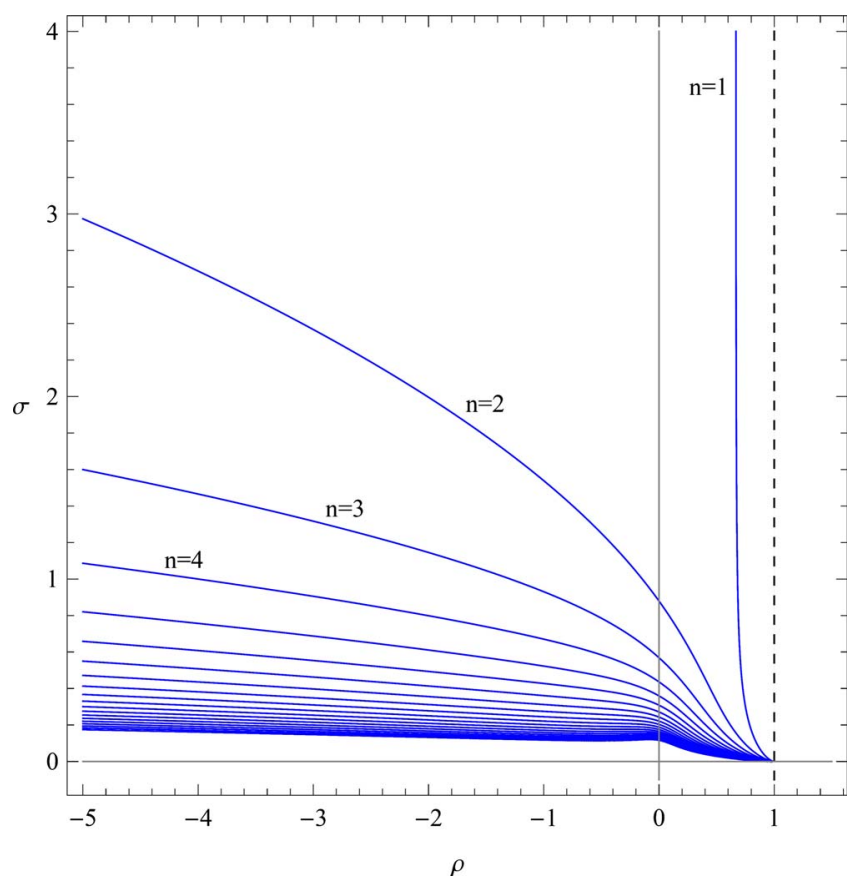

FIG. 3. (Color online) Graphs of the curves $\widetilde{C}_{n}^{-}$in the $\rho$ - $\sigma$ for $1 \leq n \leq 20$. The unmarked curves correspond to $n=5,6,7, \ldots, 20$ from top to bottom. $\widetilde{C}_{1}^{-}$has an asymptote given by $\rho \approx 0.667$. The dashed line corresponds to $\rho=1$. The solid gray lines are the coordinate axes.

Another outcome of examining the curves $\widetilde{C}_{n}^{-}$is that, in comparison to Eq. (35), the use of Eq. (34) in plotting $\widetilde{C}_{n}^{-}$ leads to substantially larger numerical errors. Therefore, in practice, it is more appropriate to employ Eq. (35) to locate the spectral singularities of the potential (2) graphically. For this reason, in preparing Fig. 3, we have used Eq. (35) to plot $\widetilde{C}_{n}^{-}$for $1 \leq n \leq 20$. This figure reveals the following facts about the curves of spectral singularities:

(i) $\widetilde{C}_{1}^{-}$has a vertical asymptote given by $\rho \approx 0.667$;

(ii) for all $n \geq 1, \widetilde{C}_{n}^{-}$is a decreasing curve;

(iii) for $n \geq 2$ the graph of $\widetilde{C}_{n}^{-}$lies above that of $\widetilde{C}_{n+1}^{-}$; and

(iv) as $n \rightarrow \infty, \widetilde{C}_{n}^{-}$tends to the $\rho$ axis [21].

\section{RESONANCE EFFECT ASSOCIATED WITH SPECTRAL SINGULARITIES}

In this section, we describe an electromagnetic waveguide that can be used to realize the resonance effect associated with the spectral singularities of the complex barrier potential [Eq. (2)].

Let $\alpha, \beta, \gamma$ be positive parameters having the dimension of length. Consider a rectangular waveguide with perfectly conducting walls that guides TE waves along the $z$ axis in the region defined by $|x|<\beta$ and $|y|<\gamma$. Suppose that the region $|z|<\alpha$ inside the waveguide is filled with an atomic gas so that the relative permittivity inside the waveguide is given by $[14,22]$

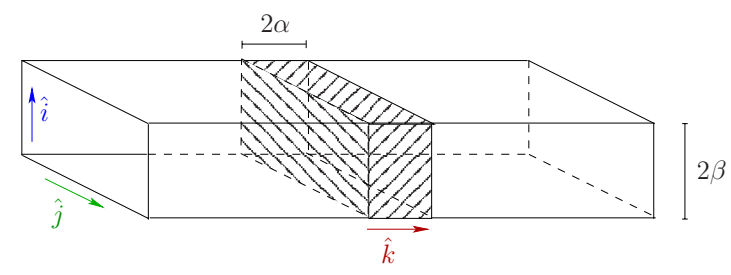

FIG. 4. (Color online) A rectangular waveguide aligned along the $z$ axis. The dashed region is filled with an atomic gas. $\hat{i}, \hat{j}$, and $\hat{k}$ are the unit vectors along the $x, y$, and $z$ axes, respectively.

$$
\varepsilon(z)= \begin{cases}1-\frac{\omega_{p}^{2}}{\omega^{2}-\omega_{0}^{2}+2 i \delta \omega} & \text { for }|z|<\alpha \\ 1 & \text { for }|z| \geq \alpha,\end{cases}
$$

where $\omega, \omega_{p}, \omega_{0}$, and $\delta$ are the frequency of the wave, the plasma frequency, the resonance frequency, and the damping constant, respectively. Figure 4 provides a schematic illustration of the waveguide.

Let $\mathfrak{K}:=\omega / c$ be the wave number, $\mathfrak{K}_{m}:=\pi m /(2 \beta)$ for every positive integer $m$, and $\phi(z)$ be a solution of

$$
\phi^{\prime \prime}(z)+\left[\mathfrak{K}^{2} \epsilon(z)-\mathfrak{K}_{m}^{2}\right] \phi(z)=0,
$$

with a continuous derivative. Then it is an easy exercise to show that the following TE wave is a solution of the Maxwell equations with appropriate boundary conditions for the above waveguide [9],

$$
\begin{aligned}
\vec{E}(\vec{r}, t)=\mathcal{E} & \operatorname{Re}\left\{e^{-i \omega t}\left\{-i \omega \sin \left[\mathfrak{K}_{m}(x+\beta)\right] \phi(z)\right\} \hat{j}\right\}, \\
\vec{B}(\vec{r}, t)= & \mathcal{E} \operatorname{Re}\left\{e ^ { - i \omega t } \left\{\sin \left[\mathfrak{K}_{m}(x+\beta)\right] \phi^{\prime}(z) \hat{i}\right.\right. \\
& \left.\left.-\mathfrak{K}_{m} \cos \left[\mathfrak{K}_{m}(x+\beta)\right] \phi(z) \hat{k}\right\}\right\} .
\end{aligned}
$$

Here $\mathcal{E}$ is a real constant and $\hat{i}, \hat{j}$, and $\hat{k}$ are the unit vectors along the $x, y$, and $z$ axes, respectively [23].

Equation (37) coincides with the eigenvalue equation,

$$
\left[-\frac{d^{2}}{d z^{2}}+v(z)\right] \phi=k^{2} \psi
$$

for the complex barrier potential [Eq. (2)] if we replace $x$ with $z$ in Eq. (2) and make the following identifications:

$$
\begin{gathered}
k=\sqrt{\mathfrak{K}^{2}-\mathfrak{K}_{m}^{2}}=\frac{\omega}{c} \sqrt{1-\frac{\Omega^{2}}{\omega^{2}}}, \quad \Omega:=\frac{\pi m c}{2 \beta}, \\
\operatorname{Re}(\mathfrak{z})=\frac{\omega^{2} \omega_{p}^{2}\left(\omega^{2}-\omega_{0}^{2}\right)}{c^{2}\left[\left(\omega^{2}-\omega_{0}^{2}\right)^{2}+4 \omega^{2} \delta^{2}\right]}, \\
\operatorname{Im}(\mathfrak{z})=\frac{-2 \omega^{3} \omega_{p}^{2} \delta}{c^{2}\left[\left(\omega^{2}-\omega_{0}^{2}\right)^{2}+4 \omega^{2} \delta^{2}\right]} .
\end{gathered}
$$

These imply 


$$
\begin{gathered}
\rho=\frac{\omega_{p}^{2}\left(\omega^{2}-\omega_{0}^{2}\right)}{\left[\left(\omega^{2}-\omega_{0}^{2}\right)^{2}+4 \omega^{2} \delta^{2}\right]\left(1-\Omega^{2} / \omega^{2}\right)}, \\
\sigma=\frac{-2 \omega \omega_{p}^{2} \delta}{\left[\left(\omega^{2}-\omega_{0}^{2}\right)^{2}+4 \omega^{2} \delta^{2}\right]\left(1-\Omega^{2} / \omega^{2}\right)} .
\end{gathered}
$$

For a TE wave given by Eqs. (38) and (39) that propagates in the waveguide, we have $\mathfrak{K}>\mathfrak{K}_{m}$, so that $k$ is real and positive. Therefore, we can use the results of Sec. III to determine the reflection and transmission amplitudes of the wave. In particular, if we can arrange the physical parameters of the system, namely, $\omega, \omega_{0}, \omega_{p}, \delta, \alpha$, and $\beta$ so that $k^{2}$ is a spectral singularity, reflection and transmission coefficients diverge and the waveguide functions as a resonator.

For the system we described above $\omega_{p}^{2}$ and $\delta$ are positive. In view of Eq. (42) this implies that $\operatorname{Im}(\mathfrak{z})<0$. Therefore, as we showed in Sec. III (Fig. 2), there is no spectral singularities. This is actually to be expected because for the physical system that we consider the presence of a spectral singularity means the emission of highly amplified electromagnetic waves. This is clearly forbidden by the law of conservation of energy. It is quite remarkable that the absence of spectral singularities for the barrier potentials with $\operatorname{Im}(\mathfrak{z}) \leq 0$, which is a purely mathematical result, can be interpreted as a direct consequence of conservation of energy in classical electrodynamics.

We can avoid the above conflict with energy conservation if we consider the situation that the sign of $\omega_{p}^{2}$ is reversed. In this case the atomic gas confined in the region $|z|<\alpha$ inside the waveguide acts as a gain medium [24]. We can achieve this by shining a laser beam along the $y$ axis to excite the resonant atoms and induce a population inversion [25]. This was also the mechanism we employed in [9] to realize the resonance effect related with the spectral singularities of the $\mathcal{P} \mathcal{T}$-symmetric barrier potential [Eq. (1)]. The main advantage of the system we consider here is that, for the cases that $\omega_{0}, \omega_{p}$, and $\delta$ are fixed, the spectral singularities occur along an infinite set of curves in the relevant parameter space, whereas for the system considered in [9] they occur at an infinite set of isolated points.

Another practical shortcoming of the system studied in [9] is that it relied on the assumption that the frequency of the propagating wave $\omega$ is equal to the resonance frequency of the gain medium $\omega_{0}$. Here we do not need to make such an assumption. This provides an additional flexibility in our attempt to adjust the parameters of the system so that the frequency of the propagating wave coincides with that of a spectral singularity. To demonstrate how we can do this, we first choose some typical values for $\omega_{0}, \omega_{p}$, and $\delta$ that determine the characteristics of the gain medium. For example, suppose that

$$
\hbar \omega_{0}=5 \mathrm{eV}, \hbar^{2} \omega_{p}^{2}=-0.04 \mathrm{eV}^{2}, \hbar \delta=1.25 \mathrm{eV} .
$$

Then $\omega_{0}$ and $\omega_{p}$ and $\delta, \rho$, and $\sigma$ become functions of $\Omega$ and $\omega$. For each choice of $\Omega$, we can select a practically attainable range of frequencies $\omega$ and plot the parametric curve $\mathcal{C}^{\Omega}$ defined by Eqs. (43) in the $\rho-\sigma$ plane for this range of values of $\omega$. The points of the intersection of $\mathcal{C}^{\Omega}$ and $\widetilde{C}_{n}^{-}$ correspond to the values of $\omega$ for which $k^{2}$ is a spectral singularity provided that Eq. (26) is also satisfied. The latter condition fixes the value of $\alpha$. The $\omega$ and $\alpha$ values obtained in this way depend on $\Omega$, the label $n$, and another discrete label $\ell_{n}$ that counts the number of times $\mathcal{C}^{\Omega}$ intersects $\widetilde{C}_{n}^{-}$as one decreases the value of $\rho$ starting from its largest allowed value, namely, 1 .

To determine the precise values of $\omega$ and $\alpha$ for a given choice of $\Omega$ and $n$, we plot the curves $\mathcal{C}^{\Omega}$ and $\widetilde{C}_{n}^{-}$in the $\rho$ - $\sigma$ plane as in Fig. 5 and select an intersection point, i.e., fix $\ell_{n}$. We then determine the coordinates $\left(\rho_{\star}, \sigma_{\star}\right)$ of this point graphically [26]. Next, we numerically solve for $\omega$ in

$$
\rho=\rho_{\star}, \quad \sigma=\sigma_{\star},
$$

where the left-hand side of these equations is given by Eqs. (43). A consistency check on this calculation is that both of these equations must give the same value for $\omega$. Having obtained $\omega$, we compute $k$ using Eq. (40), substitute this value of $k$ and Eq. (45) in Eq. (26), and solve for $\alpha$ in the resulting equation.

Figure 5 shows the plots of the curves $\mathcal{C}^{\Omega}$ and $\widetilde{C}_{n}^{-}$for various values of $\Omega$ and $n$. For every $\Omega$, the curve $\mathcal{C}^{\Omega}$ intersects each of the curves $\widetilde{C}_{n}^{-}$at least once and at most three times, i.e., $1 \leq \ell_{n} \leq 3$. As one adopts sufficiently large values for $\beta / m$ (sufficiently small values for $\Omega$ ), the number of intersection points increases. This number equals to the number of different frequencies $\omega$ at which a spectral singularity occurs. Note, however, that each of these spectral singularities corresponds to a different value of the parameter $\alpha$. Furthermore, because for each value of $\Omega, \mathcal{C}^{\Omega}$ intersects $\widetilde{C}_{n}^{-}$for all $n$, for every value of $\beta / m$ there is an infinity of choices for the $\omega$ (and $\alpha$ ) values that yield a spectral singularity.

It turns out that for each value of $\beta / m$, one can select $n$ so that the related spectral singularities correspond to experimentally preferable values for $\alpha$ and $\omega$. For example, for the principal mode $(m=1)$ of a waveguide of height $2 \beta=1 \mathrm{~cm}$, choosing $n=10000$ and $\ell_{n}=2$, we find a spectral singularity of frequency $\omega \approx 2.1555 \mathrm{eV} / \hbar$ (wavelength: $\lambda$ $\approx 575.20 \mathrm{~nm}$ ) provided that we maintain an active region of length $2 \alpha \approx 2.8786 \mathrm{~mm}$. For future reference we will use * to label this spectral singularity. In fact, as we would expect, changing the value of $n$ starting from such a large initial value, we find a large number of closely spaced spectral singularities all belonging to the experimentally attainable range of values of $\alpha$ and $\omega$.

Figure 6 shows the location of the spectral singularities for $2 \beta / m=1 \mathrm{~cm}$ that we obtained for $10000 \leq n \leq 11000$ and $\ell_{n}=2,3$. For these choices of $n$, the spectral singularities associated with $\ell_{n}=1$ correspond to extremely large (experimentally unattainable) values for $2 \alpha$. As suggested by Fig. 6 , using a waveguide of height $2 \beta=1 \mathrm{~cm}$ and adjusting the length $2 \alpha$ of the gain region (in the millimeter range), we can generate spectral singularities at almost every point of the visible spectrum or outside it. This is also supported by a detailed examination of other choices for the values of $2 \beta$ and $n$ that we do not fully report here except for a few illustrative cases that we include in Tables I and II. 

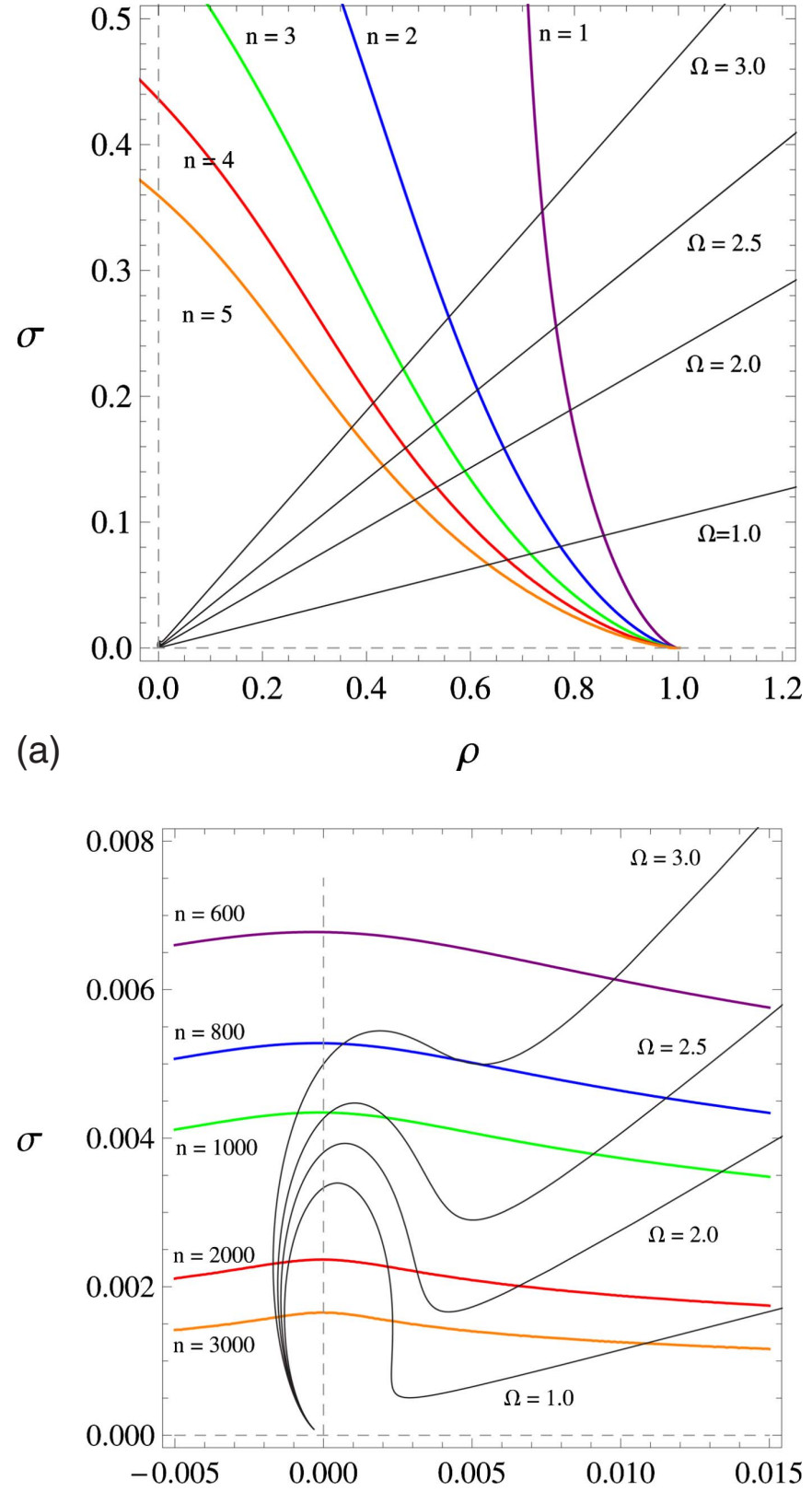

(b)

FIG. 5. (Color online) Graphs of $\mathcal{C}^{\Omega}$ (thin black curves) and $\widetilde{C}_{n}^{-}$(thick colored curves) for $\omega_{0}, \omega_{p}, \delta$ as given by Eq. (44) and $\Omega=1.0,2.0,2.5,3.0 \mathrm{eV} / \hbar$ that correspond to $\beta / \mathrm{m}$ $=310.0,155.0,124.0,103.3 \mathrm{~nm}$, respectively. The top figure shows the intersection points of $\mathcal{C}^{\Omega}$ and $\widetilde{C}_{n}^{-}$with $n=1,2,3,4,5$. These correspond to $\ell_{n}=1$. The bottom figure shows the intersection points of $\mathcal{C}^{\Omega}$ and $\widetilde{C}_{n}^{-}$with $n=600,800,1000,2000,3000$. For these points $\ell_{n}=1,2$, or 3 .

As shown in Fig. 6 and Tables I and II, for $\ell_{n}=2$ the wavelength of spectral singularity is an increasing function of the length $2 \alpha$, while for $\ell_{n}=3$ it is a decreasing function of $2 \alpha$. This shows the availability of a wide range of values of parameters of the system that lead to a spectral singularity.

According to Table I, for $n=10000$ and $\ell_{n}=2$, both of the choices $2 \beta / m=1 \mathrm{~mm}$ and $2 \beta / m=1 \mathrm{~cm}$ give rise to spectral
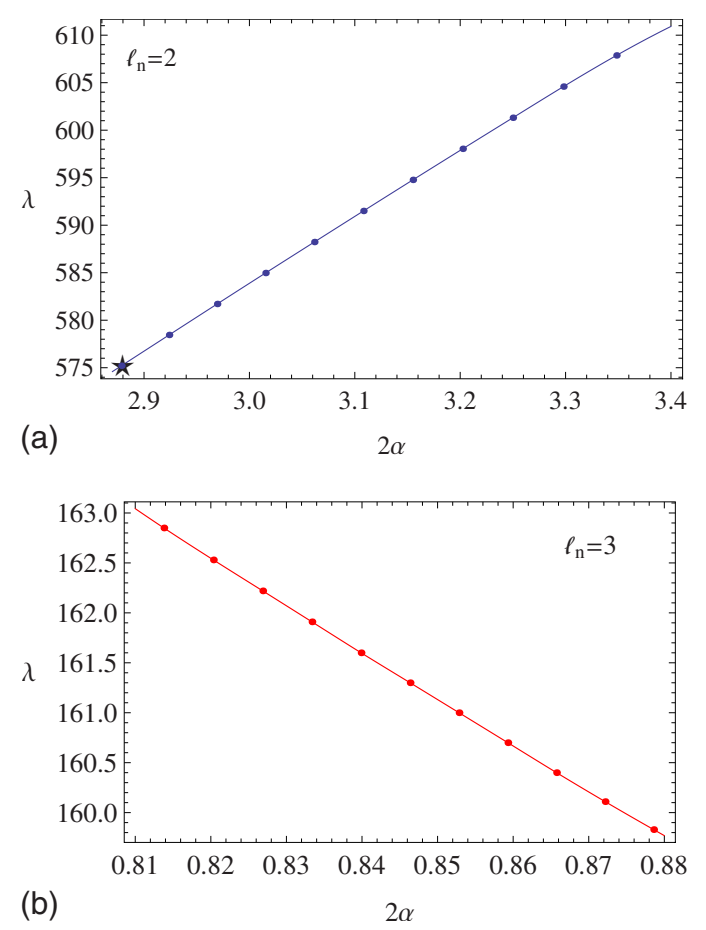

FIG. 6. (Color online) Location of the spectral singularities in the $2 \alpha-\lambda$ plane for $2 \beta / m=1 \mathrm{~cm}$ and $10000 \leq n \leq 11000$. The numerical values of $2 \alpha$ (length of the gain region) and $\lambda$ (wavelength of the emitted wave) are given in $\mathrm{mm}$ and $\mathrm{nm}$, respectively. The displayed dots represent the spectral singularities with $n$ $=10000,10100,10200, \ldots, 11000$ from the left to the right. The curve segments joining adjacent dots represent 99 isolated points each corresponding to a different spectral singularity. The top and bottom figures show the spectral singularities with $\ell_{n}=2$ and $\ell_{n}$ $=3$, respectively. The spectral singularity $\star$ is also displayed.

singularities with the same values for the wavelength $\lambda$, the length parameter $2 \alpha$, and the complex refractive index $\sqrt{\varepsilon}$. Therefore, to observe this spectral singularity one does not need to fine tune the height of the waveguide. This is also true for $n \gtrsim 2000$ and $\ell_{n}=3$.

Figure 7 shows the graphs of $\log _{10}\left(|T|^{2}+|R|^{2}\right)$ as a function of $\omega / \omega_{s}$ where $\omega_{s}$ is the frequency of the spectral singularity $\star$ with $n=10000$ [27]. This is represented by the peak at $\omega / \omega_{s}=1$ that corresponds to an amplification of the emitted electromagnetic energy by a factor of $\geq 10^{15}$. We can increase this factor if we use more precise values for the $\omega$ and $2 \alpha$ parameters associated with $\star$. The other peaks represent the spectral singularities with $n=10001,9999$, $10002,9998, \ldots$ and $\ell_{n}=2$. These are lower than the central peak because the corresponding spectral singularities have a slightly different values for $\omega$ and $2 \alpha$ than those for $\star$ that we have adopted in preparing this figure. The location of the peaks can be easily explained. Because to a good approxmation the frequency of the spectral singularities with $n$ $\approx 10000$ and $\ell_{n}=2$ decreases as a linear function of $n$ (see Fig. 6), changing $n$ by one unit, i.e., by a factor of $10^{-4}$, leads to a change in $\omega$ by the same factor. This explains the occurrence of the peaks at the $\omega / \omega_{s}$ values that differ from unity by an integer multiple of $10^{-4}$. Another interesting observation is that the amplification effect persists even if we change 
TABLE I. The wavelength $\lambda$ of the TE wave and the length $2 \alpha$ and complex refractive index $\sqrt{\varepsilon}$ of the gain region corresponding to a spectral singularity with $\omega_{p}, \omega_{0}, \delta$ given by Eq. (44) and $n=10000$. The boldface figures are those of the spectral singularity $\star$.

\begin{tabular}{|c|c|c|c|}
\hline \multicolumn{4}{|c|}{$2 \beta / m=1.0000 \mu \mathrm{m}$} \\
\hline$\ell_{n}$ & $\lambda$ & $2 \alpha$ & $\sqrt{\varepsilon}$ \\
\hline 1 & $1.6798 \mu \mathrm{m}$ & $15.517 \mathrm{~mm}$ & $0.99919-6.1408 \times 10^{-5} i$ \\
\hline 2 & $614.03 \mathrm{~nm}$ & $3.2291 \mathrm{~mm}$ & $0.99910-2.1814 \times 10^{-4} i$ \\
\hline 3 & $162.61 \mathrm{~nm}$ & $0.81531 \mathrm{~mm}$ & $1.00045-2.6078 \times 10^{-4} i$ \\
\hline \multicolumn{4}{|c|}{$2 \beta / m=1.0000 \mathrm{~mm}$} \\
\hline$\ell_{n}$ & $\lambda$ & $2 \alpha$ & $\sqrt{\varepsilon}$ \\
\hline 1 & $1.9974 \mathrm{~mm}$ & $307.845 \mathrm{~m}$ & $0.99920-4.9699 \times 10^{-8} i$ \\
\hline 2 & $575.20 \mathrm{~nm}$ & $2.8786 \mathrm{~mm}$ & $0.99908-2.4333 \times 10^{-4} i$ \\
\hline 3 & $162.85 \mathrm{~nm}$ & $0.81379 \mathrm{~mm}$ & $1.00045-2.6259 \times 10^{-4} i$ \\
\hline \multicolumn{4}{|c|}{$2 \beta / m=1.0000 \mathrm{~cm}$} \\
\hline$\ell_{n}$ & $\lambda$ & $2 \alpha$ & $\sqrt{\varepsilon}$ \\
\hline 1 & $1.9982 \mathrm{~cm}$ & $6.7894 \mathrm{~km}$ & $0.99920-4.968 \times 10^{-9} i$ \\
\hline 2 & $575.20 \mathrm{~nm}$ & $2.8786 \mathrm{~mm}$ & $0.99908-2.4333 \times 10^{-4} i$ \\
\hline 3 & $162.84 \mathrm{~nm}$ & $0.81379 \mathrm{~mm}$ & $1.00045-2.6259 \times 10^{-4} i$ \\
\hline
\end{tabular}

$\omega$ by as much as $10 \%$ (see the last graph depicted in Fig. 7.) This is another indication of the suitability of the proposed setup for an experimental realization of the spectral singularity-related resonance effect.

\section{CONCLUSION}

Spectral singularities are the energies of a rather strange type of scattering states that similarly to resonance states have infinite reflection and transmission coefficients. Physically this means that if one arranges to scatter a plane wave

TABLE II. The wavelength $\lambda$ of the TE wave and the length $2 \alpha$ and complex refractive index $\sqrt{\varepsilon}$ of the gain region corresponding to a spectral singularity with $\omega_{p}, \omega_{0}, \delta$ given by Eq. (44), $2 \beta / m$ $=1.0000 \mathrm{~cm}, \ell_{n}=2,3$, and $n=2000,3000,4000,5000$.

\begin{tabular}{lccc}
\hline \hline & $\begin{array}{c}\lambda \\
n\end{array}$ & $\begin{array}{c}2 \alpha \\
(\mathrm{mm})\end{array}$ & $\sqrt{\varepsilon}$ \\
\hline \multicolumn{3}{c}{$\ell_{n}=2$} \\
2000 & 306.59 & 0.30685 & $0.99902-1.1437 \times 10^{-3} i$ \\
3000 & 347.47 & 0.52173 & $0.99893-7.763 \times 10^{-4} i$ \\
4000 & 382.28 & 0.76534 & $0.99895-5.8934 \times 10^{-4} i$ \\
5000 & 415.09 & 1.03877 & $0.99897-4.757 \times 10^{-4} i$ \\
& & & \\
& & $\ell_{n}=3$ & \\
2000 & 220.78 & 0.22059 & $1.00055-1.1701 \times 10^{-3} i$ \\
3000 & 203.54 & 0.30504 & $1.00064-8.043 \times 10^{-4} i$ \\
4000 & 193.10 & 0.38589 & $1.00062-6.1592 \times 10^{-4} i$ \\
5000 & 185.45 & 0.46327 & $1.00059-5.006 \times 10^{-4} i$ \\
\hline \hline
\end{tabular}
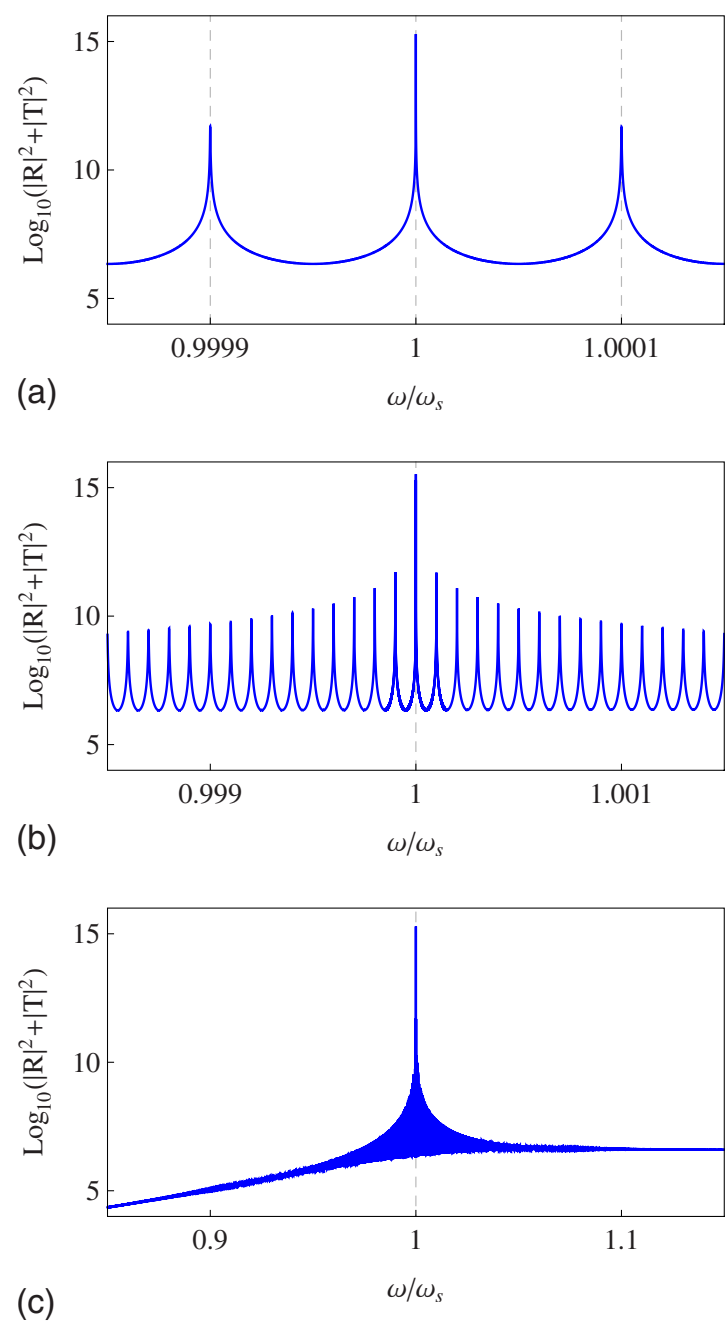

FIG. 7. (Color online) Graphs of $\log _{10}\left(|T|^{2}+|R|^{2}\right)$ as a function of $\omega / \omega_{s}$ for three different ranges of values of $\omega / \omega_{s} \cdot|R|^{2}$ and $|T|^{2}$ are the reflection and transmission coefficients (see endnote in [27]). $\omega_{s}=2.15548 \mathrm{eV} / \hbar$ is the frequency of the spectral singularity $\star$ that occurs for $\omega_{p}, \omega_{0}, \delta$ given by Eq. (44), $n=10000, \ell_{n}=2$, $2 \underline{\beta} / \mathrm{m}=1.00000 \mathrm{~cm}, 2 \alpha=2.878644 \mathrm{~mm}, \lambda=575.2046 \mathrm{~nm}$, and $\sqrt{\varepsilon}=0.999081358-0.000243330 i$. The highest peak represents $\star$. The other peaks correspond to the spectral singularities with $n=10001,9999,10002,9998, \ldots$.

of energy close to that of a spectral singularity, the system amplifies the wave enormously. Therefore, spectral singularities are related to a novel resonance phenomenon that awaits experimental verification.

For a real potential, the unitarity of dynamics implies that the reflection and transmission coefficients add up to unity. Therefore, spectral singularities cannot arise for a real potential. In this paper, we have explored the spectral singularities of a complex barrier potential and showed how this potential enters the description of the electromagnetic wave propagation in certain waveguides. If we adjust the physical parameters of the waveguide system so that the frequency of the incoming wave is close to that of a spectral singularity, the waveguide emits a substantially amplified wave of the same frequency from both ends. We expect this phenomenon to find useful applications in optics particularly because we can 
adjust the resonance frequency to attain any desired value.

A remarkable result of our investigation is that spectral singularities of the complex barrier potential occur only for certain values of the coupling constant with positive imaginary part. While the mathematical reason for this phenomenon is obscure, it has a simple physical justification. The condition that the coupling constant has a positive imaginary part corresponds to the case that the waveguide includes a gain region. This is necessary for the existence of the resonance effect because of the law of conservation of energy.

One of the main goals of the present investigation was to examine the range of critical values of the physical parameters of the waveguide system that corresponded to the occurrence of spectral singularities. We showed that indeed these parameters spanned a very large spectrum of values.
This makes the waveguide system we considered in this paper a plausible candidate for observing the spectral singularity-related resonance effect.

\section{ACKNOWLEDGMENTS}

I wish to thank Hossein Mehri-Dehnavi and Ali Serpengüzel for reading the first draft of this paper and making useful comments. I am also indebted to Alphan Sennaroğlu and Aref Mostafazadeh for illuminating discussions. This work has been supported by the Scientific and Technological Research Council of Turkey (TÜBITAK) in the framework under Project No. 108 T009 and by the Turkish Academy of Sciences (TÜBA).
[1] A. Mostafazadeh, e-print arXiv:0810.5643.

[2] A. Mostafazadeh, J. Phys. A 39, 10171 (2006).

[3] W. D. Heiss, J. Phys. A 37, 2455 (2004).

[4] M. A. Naimark, Trudy Moskow Mat. Obshch. 3, 181 (1954) [Am. Math. Soc. Transl. 16, 103 (1960).]

[5] W. D. Heiss, Phys. Rep. 242, 443 (1994); E. Narevicius and N. Moiseyev, Phys. Rev. Lett. 81, 2221 (1998); 84, 1681 (2000); M. V. Berry, J. Mod. Opt. 50, 63 (2003); Czech. J. Phys. 54, 1039 (2004); A. A. Mailybaev, O. N. Kirillov, and A. P. Seyranian, Phys. Rev. A 72, 014104 (2005); M. Müller and I. Rotter, J. Phys. A 41, 244018 (2008); H. Mehri-Dehnavi and A. Mostafazadeh, J. Math. Phys. 49, 082105 (2008); S. Klaiman, U. Günther, and N. Moiseyev, Phys. Rev. Lett. 101, 080402 (2008).

[6] C. Dembowski, H.-D. Gräf, H. L. Harney, A. Heine, W. D. Heiss, H. Rehfeld, and A. Richter, Phys. Rev. Lett. 86, 787 (2001); C. Dembowski, B. Dietz, H.-D. Gräf, H. L. Harney, A. Heine, W. D. Heiss, and A. Richter, Phys. Rev. E 69, 056216 (2004); T. Stehmann, W. D. Heiss, and F. G. Scholtz, J. Phys. A 37, 7813 (2004).

[7] Here we only consider Hamiltonians of the form $H=-\left(d^{2} /\right.$ $\left.d x^{2}\right)+v(x)$, where $x$ is real and $v(x)$ is a complex scattering potential.

[8] A. Mostafazadeh and H. Mehri-Dehnavi, J. Phys. A 42, 125303 (2009).

[9] A. Mostafazadeh, Phys. Rev. Lett. 102, 220402 (2009).

[10] B. F. Samsonov, J. Phys. A 38, L397 (2005); 38, L571 (2005).

[11] M. A. Naimark, Linear Differential Operators, Pt. II (Ungar, New York, 1968).

[12] A. Mostafazadeh, J. Phys. A 39, 13495 (2006).

$[13] \operatorname{sgn}(x):=x /|x|$ if $x \neq 0$ and $\operatorname{sgn}(0):=0$.

[14] A. Ruschhaupt, F. Delgado, and J. G. Muga, J. Phys. A 38, L171 (2005).
[15] J. G. Muga, J. P. Palao, B. Navarro, and I. L. Egusquiza, Phys. Rep. 395, 357 (2004).

[16] R. R. D. Kemp, Can. J. Math. 10, 447 (1958); J. Schwartz, Commun. Pure Appl. Math. 13, 609 (1960); V. É. Ljance, Mat. Sb. 64, 521 (1964) [Am. Math. Soc. Transl. 60, 185 (1967)]; 65, 47 (1964) [Am. Math. Soc. Transl. 60, 227 (1967)].

[17] G. Sh. Guseinov, Pramana, J. Phys. 73, 587 (2009).

[18] A. J. F. Siegert, Phys. Rev. 56, 750 (1939).

[19] We use $\sqrt{1-\mathfrak{z} / k^{2}}$ to denote the principal value of $\left(1-\mathfrak{z} / k^{2}\right)^{1 / 2}$ whose argument belongs to $[0, \pi)$.

[20] For example, typical numerical schemes will produce $k$ values with a small imaginary part. These do not correspond to scattering states and must therefore be rejected.

[21] This can be easily shown using Eq. (31).

[22] J. D. Jackson, Classical Electrodynamics (Wiley, New York, 1975).

[23] Note that we use physicists' convention of expressing the timeharmonic part of the wave as $e^{-i \omega t}$ as opposed to engineers' convention that uses $e^{i \omega t}$. This leads to a change in sign in the imaginary part of permittivity (and complex refractive index).

[24] A. Yariv and P. Yeh, Photonics (Oxford University Press, Oxford, 2007).

[25] $\omega_{p}^{2}$ is proportional to the difference between the number density of atoms in the ground and excited states which becomes negative due to population inversion [24].

[26] Using MATHEMATICA and without making any special efforts we could zoom in the neighborhood of the relevant intersection point and obtain $\rho_{\star}$ and $\sigma_{\star}$ up to six significant digits for various values of $\Omega, n$, and $\ell_{n}$.

[27] Because of the symmetry of the problem, and as we can easily verify by inserting Eq. (9) in Eq. (6), the left and right reflection (and transmission) coefficients coincide: $R^{r}=R^{l}=: R$ and $T^{r}=T^{l}=: T$. 